\title{
Rhinoplasty for Saddle Nose with Sarcoidosis
}

\author{
Masaki Yazawa1 ${ }^{*}$, Hayato Nagashima1, Katsuya Suzuki², Kazuo Kishi1 \\ ${ }^{1}$ Department of Plastic and Reconstructive Surgery, Keio University School of Medicine, Tokyo, Japan \\ ${ }^{2}$ Division of Rheumatology, Department of Internal Medicine, Keio University School of Medicine, Tokyo, Japan \\ Email: ${ }^{*}$ prsyazawa@gmail.com
}

Received 28 April 2015; accepted 31 May 2015; published 3 June 2015

Copyright (C) 2015 by authors and Scientific Research Publishing Inc.

This work is licensed under the Creative Commons Attribution International License (CC BY). http://creativecommons.org/licenses/by/4.0/

(c) () Open Access

\begin{abstract}
Background: Cases of acquired saddle nose have been reported; however, reports of sarcoidosis with acquired saddle nose are very rare. The main cause of sarcoidosis is estimated to be an abnormal immune reaction rather than infection. Aim: Rhinoplasty using an autologous rib was planned and performed without plate fixation. Case Presentation: Our patient was diagnosed with sarcoidosis at the age of 25 years. She developed nasal congestion and rhinitis at the age of 42 years. Her nose began to show the saddle nose deformity at the age of 43 years. A rhinoplasty using an autologous rib was performed without plate fixation. Conclusion: After the operation, her nose maintained a favorable shape without sarcoidosis worsening. This use of rhinoplasty for treating saddle nose possibly prevents the worsening of sarcoidosis.
\end{abstract}

\section{Keywords}

Saddle Nose, Sarcoidosis, Rhinplasty

\section{Introduction}

Saddle nose deformity has both congenital and acquired causes. Excluding congenital diseases, diseases that manifest saddle nose include recurrent multiple chondritis, granulomatosis with polyangitis [1], morbus, Hansen's disease, Crohn disease [2], and traumatic injury [3]. However, saddle nose with sarcoidosis is unsolvable and reports are very rare [4]-[6]. The main cause of sarcoidosis is estimated to be an abnormal immune reaction rather than infection [7]. As such, the use of surgical procedures and the presence of persistent inflammation can result in its progression. Accordingly, rhinoplasty using an autologous rib [8] [9] was planned and performed without plate fixation.

\footnotetext{
"Corresponding author.
} 
This use of rhinoplasty for treating saddle nose possibly prevents the worsening of sarcoidosis and maintained a favorable shape in nose.

\section{Case Presentation}

Transbronchial lung biopsy (TBLB) performed when the patient was 25 showed epithelioid cell granuloma that was confirmed as sarcoidosis. Traveling infiltration shadows in the lung were followed closely without treatment. She developed nasal congestion and rhinitis at the age of 42 years. Her nose began to show the saddle nose deformity at the age of 43 years. Secondary recurrent multiple chondritis was suspected and steroid hormone therapy was administered. Consecutive close investigations confirmed that the diagnosis was not established without sarcoidosis. Her nose deformity progressed and rhinoplasty was planned. Preoperative computed tomography showed that the nasal bone was intact. Preoperative magnetic resonance imaging showed that the alar cartilages were intact but the nasal septum was atrophied and absorbed (Figure 1). Our patient was 45 years of age at the time of surgery (Figure 2).

\section{Operation}

Open rhinoplasty (rim incision and a columellanasi V-shape incision) was selected. Her right eighth rib bone and rib cartilage were harvested with the periosteum and perichondrium. The rib cartilage was shaped into a semisphere and used asthe nasal tip. A strut (40 mm long, $3 \mathrm{~mm}$ wide) was carved from the inferior side of the rib bone for the nasal crest and was made of cortical bone. A hole was created in the rib bone near the cartilage junction to insert the strut. The anterior nasal spine (ANS) was revealed using a 2-cm incision in the oral vestibule and shaped as a base of the strut. The strut was set on the base of the ANS and connected to the nasal crest bone by insertion through the columella tissue. Perichondrium was used to cover the nasal tip cartilage and affix the rib bone to the strut (Figure 3). No extra fixation was needed, for example, by plating or wiring. The skin of the nasal tip presented no problems postoperatively (Figure 4).

\section{Discussion}

Excluding congenital diseases, diseases thatmanifest saddle nose include recurrent multiple chondritis, granulomatosis with polyangitis [1], morbus, Hansen's disease, Crohn disease [2], and traumatic injury [3]. However, cases of saddle nose with sarcoidosis are very rare [4]-[6]. In cases of sarcoidosis, highly invasive surgical procedures and persistent inflammationcan contribute to its progression. As such, rhinoplasty with autologous tissue was planned except suture thread. To ensure stable fixation of the grafted rib to the nasal bone, the ventral side

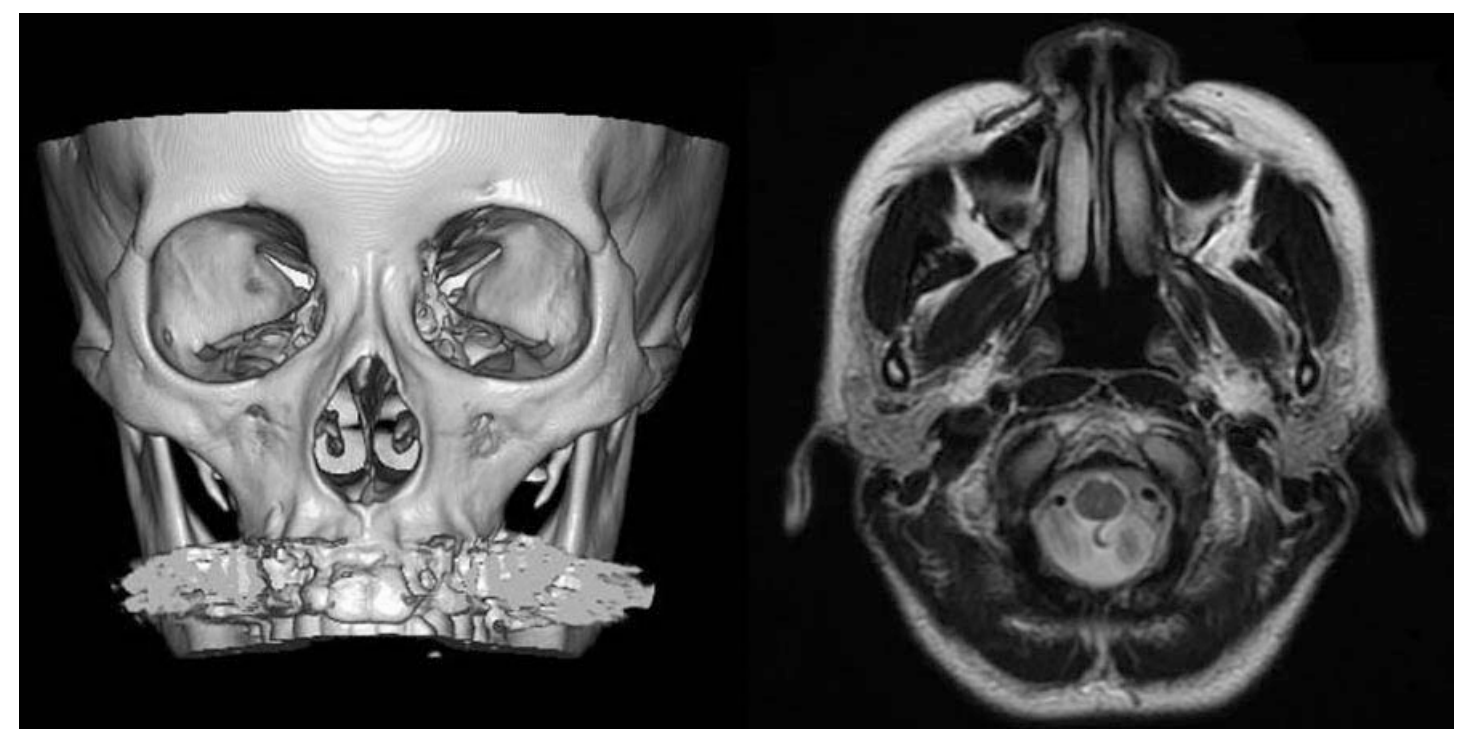

Figure 1. Preoperative computed tomography showed that the nasal bone was intact (left). Preoperative magnetic resonance imaging showed that the alar cartilages were intact but the nasal septum was atrophied and absorbed (right). 


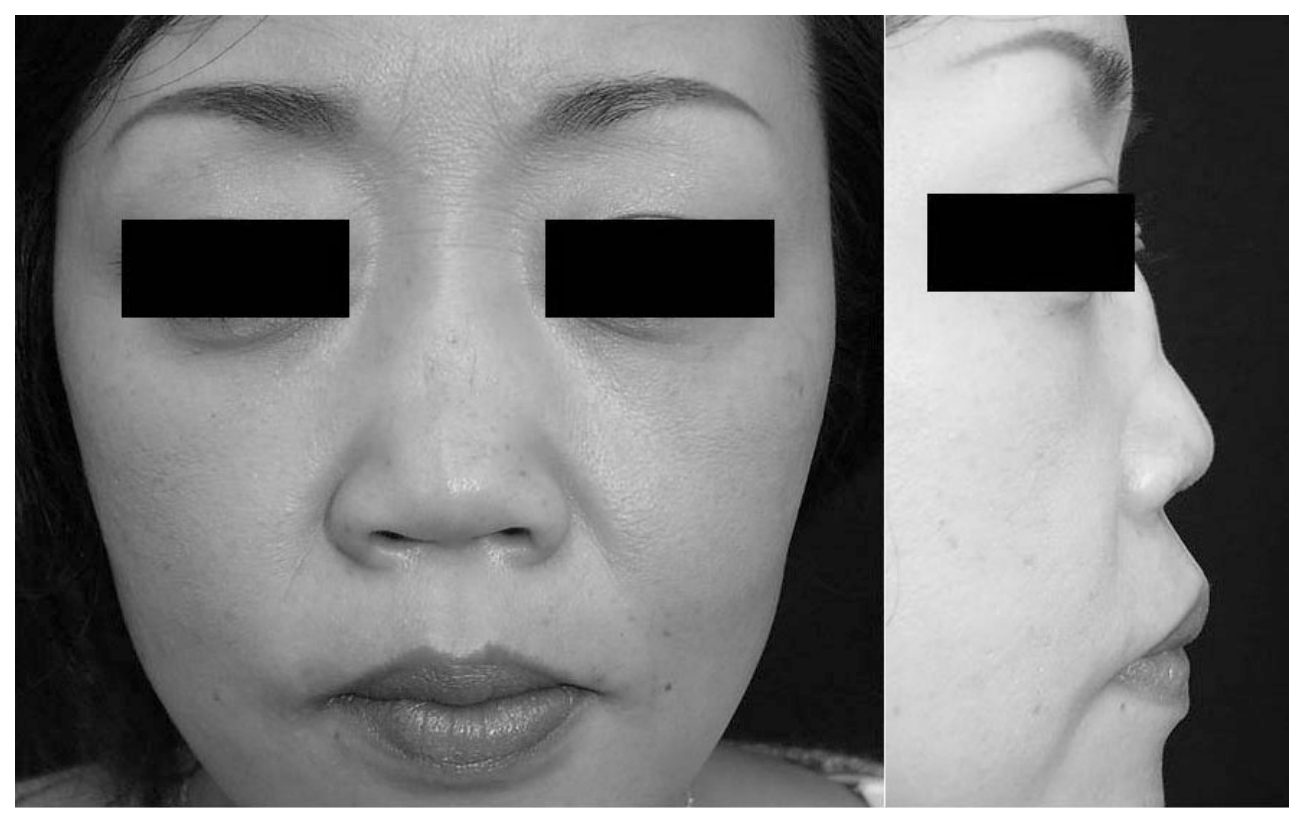

Figure 2. Preoperative view.

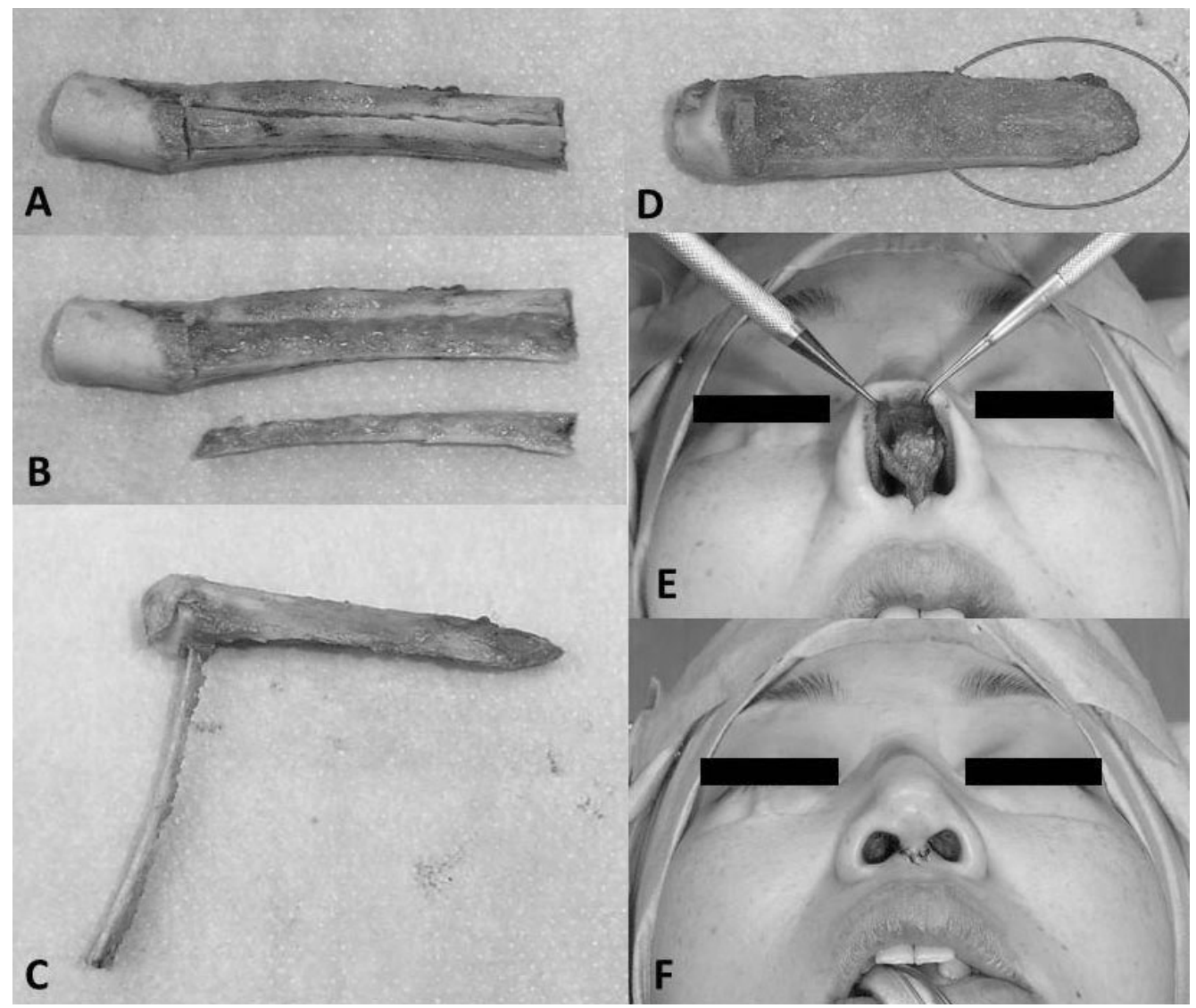

Figure 3. Intraoperative view (A) autologous right eighth rib bone and rib cartilage; (B) A strut (40 mm long, $3 \mathrm{~mm}$ wide) was carved from the inferior side of the rib bone; (C) A hole was created in the rib bone near the cartilage junction to insert the strut; (D) The ventral side of the rib was carved into a concave shape (red circle) to fit the nasal bone surface; (E) Perichondrium was used to cover the nasal tip cartilage and affix the rib bone to the strut; (F) No extra fixation was needed, for example, by plating or wiring. 
of the rib was carved into a concave shape to fit thenasal bone surface, and the rib was inserted under the periosteum of the nasal bone. The cartilage at the nasal tip was covered with perichondrium that was sutured to the base of thecolumella. This perichondrium fixation decreased the pressure overload of the grafted rib bone against the nasal skin tip and prevented the bone from being exposed to the nasal tip skin (Figure 5). Additionally, in cases of nasal tip skin necrosis, the cartilage is not readily exposed through the perichondrium. The

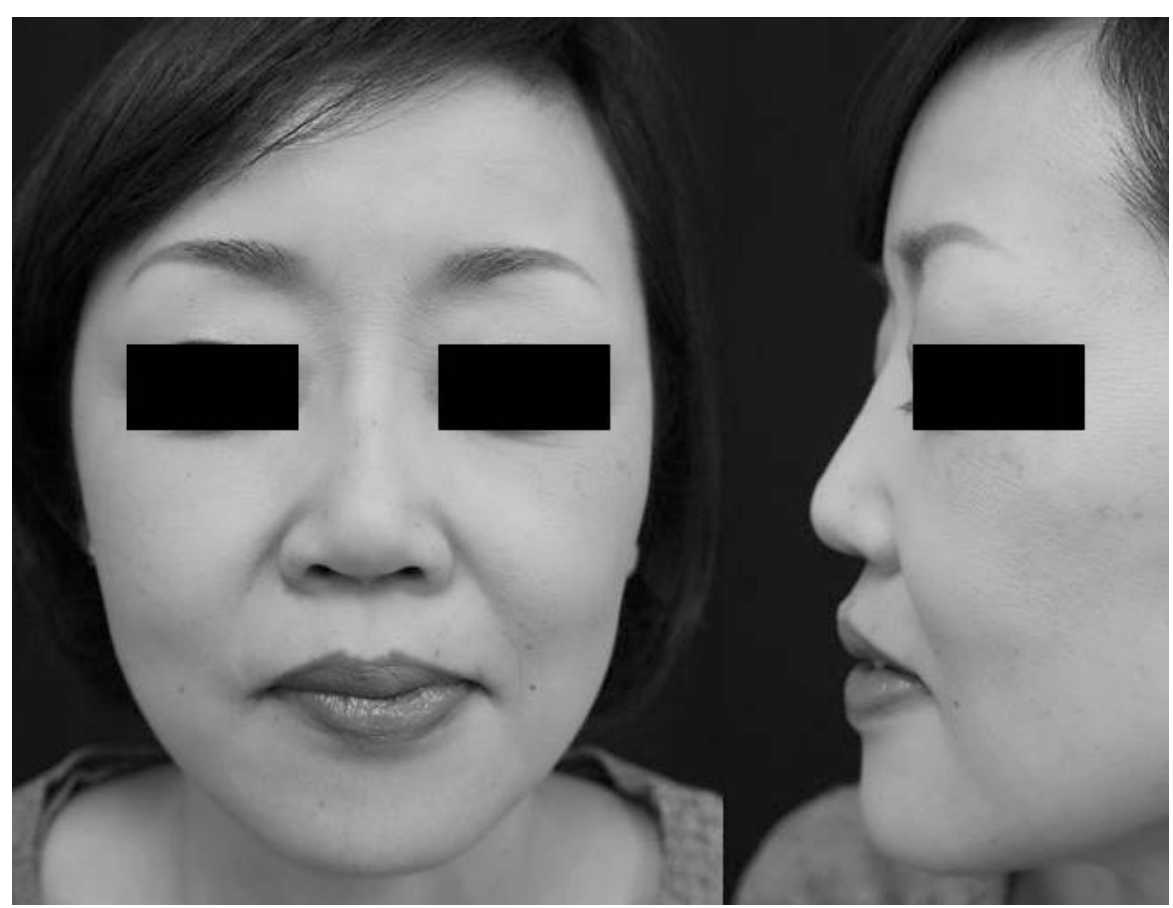

Figure 4. Two-year postoperative view.

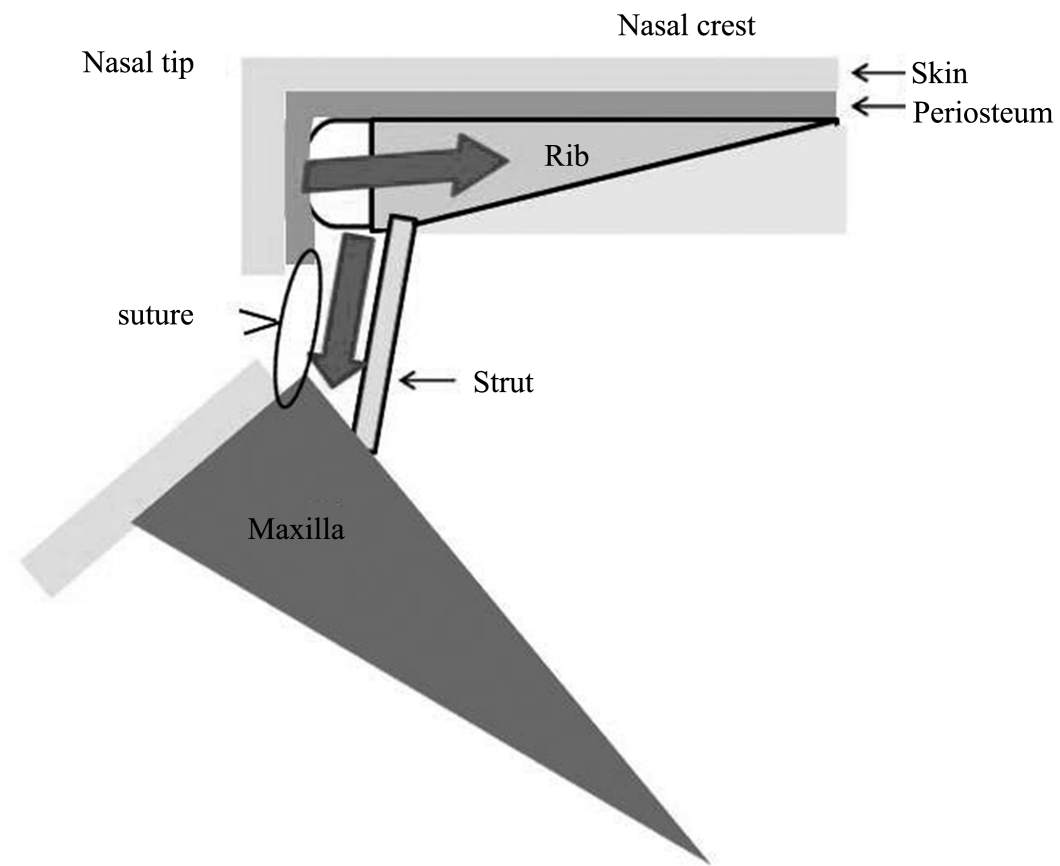

Figure 5. This perichondrium fixation decreased the pressure overload of the grafted rib bone against the nasal skin tip and prevented the bone from being exposedto the nasal tip skin. 
covered cartilage with perichondrium is easily packed with granulation and epithelializes quickly. Pathological analysis ofthe tissue around the nasal septum showed that the tissue consisted of adipose and connective tissue but no cartilaginous tissue. The tissues had a low level of inflammatory cell invasion with slight nonspecific inflammation with no evidence of angiitisor granuloma formation.

Various genetic and environmental factors have been etiologically suggested as mechanisms of sarcoidosis [10]. Propionibacterium acnes is one of the earliest proposed infectious agents. This bacterium is recently reported to be a factor for granuloma formation in sarcoidosis [11]. In this case unfortunately, pathological analysis does not show evidence of granuloma formation. However, in our patient, TBLB-induced granuloma formation has revealed the presence of $P$. acnes by PAB antibody stain at the age of 44 years. As such, these results are interesting in our attempt to clarify the mechanism of progressive saddle nose deformity.

Cases of saddle nose with sarcoidosis are very rare. This use of rhinoplasty for treating saddle nose possibly prevents the worsening of sarcoidosis.

\section{Ethical Concerns}

None.

\section{Funding}

None.

\section{Conflict of Interest}

None.

\section{Acknowledgements}

None.

\section{References}

[1] Vogt, P.M., Gohritz, A., Haubitz, M., et al. (2011) Reconstruction of Nasal Deformity in Wegener’s Granulomatosis: Contraindication or Benefit? Aesthetic Plastic Surgery, 35, 156-161. http://dx.doi.org/10.1007/s00266-010-9568-9

[2] Merkonidis, C., Verma, S. and Salam, M.A. (2005) Saddle Nose Deformity in a Patient with Crohn's Disease. The Journal of Laryngology \& Otology, 119, 573-576. http://dx.doi.org/10.1258/0022215054352126

[3] Sachse, F. and Stoll, W. (2010) Nasal Surgery in Patients with Systemic Disorders. Head and Neck Surgery, 9.

[4] Shipchandler, T.Z., Chung, B.J. and Alam, D.S. (2008) Saddle Nose Deformity Reconstruction with a Split Calvarial Bone L-Shaped Strut. Archives of Facial Plastic Surgery, 10, 305-311. http://dx.doi.org/10.1001/archfaci.10.5.305

[5] Gurkov, R. and Berghaus, A. (2009) Nasal Reconstruction in Advanced Sinunasal Sarcoidosis. Rhinology, 47, 327329.

[6] Colden, D.G., Busaba, N.Y. (2000) Sarcoidosis Presenting as Recurrent Nasal Polyps. Otolaryngology—Head and Neck Surgery, 123, 519-521. http://dx.doi.org/10.1067/mhn.2000.106193

[7] Valeyre, D., Prasse, A., Nunes, H., et al. (2014) Sarcoidosis. Lancet, 383, 1155-1167. http://dx.doi.org/10.1016/S0140-6736(13)60680-7

[8] Pribitkin, E.A., Ezzat, W.H. (2009) Classification and Treatment of the Saddle Nose Deformity. Otolaryngologic Clinics of North America, 42, 437-461. http://dx.doi.org/10.1016/j.otc.2009.03.004

[9] Isac, C., Mihajlovic, D., Bratu, T., et al. (2012) Severe Saddle Nose Deformity Reconstruction with Rib Cartilage. Chirurgia, 107, 809-815.

[10] Costabel, U. and Hunninghake, G.W. (1999) ATS/ERS/WASOG Statement on Sarcoidosis. Sarcoidosis Statement Committee. American Thoracic Society. European Respiratory Society. World Association for Sarcoidosis and Other Granulomatous Disorders. European Respiratory Journal, 14, 735-737. http://dx.doi.org/10.1034/j.1399-3003.1999.14d02.x

[11] Negi, M., Takemura, T., Guzman, J., et al. (2012) Localization of Propionibacterium acnes in Granulomas Supports a Possible Etiologic Link between Sarcoidosis and the Bacterium. Modern Pathology, 25, 1284-1297. http://dx.doi.org/10.1038/modpathol.2012.80 